\title{
On a Certain Series of Abel
}

\author{
By J. Cuunie
}

(Received 20th November, 1952.)

1. Introduction. If we express $e^{z \lambda}$, qua function of $\lambda$, in terms of $\lambda e^{\lambda}$ by Langrange's theorem we find, for $|\lambda|$ sufficiently small,

$$
e^{z \lambda}=\sum_{0}^{\infty} \frac{z(z-n)^{n \sim 1}}{n !}\left(\lambda e^{\lambda}\right)^{n} .
$$

If we now regard $\lambda$ as a parameter and $z$ as the independent variable then (1.1) can be written

$$
e^{z \lambda}=\sum_{0}^{\infty} \frac{z(z-n)^{n-1}}{n !}\left[\frac{d^{n}}{d z^{n}} e^{z \backslash}\right]_{z=n} .
$$

Supposing, therefore, that a function is of the form $f(z)=\int e^{z \lambda} \phi(\lambda) d \lambda$, then

$$
f(z)=\sum_{0}^{\infty} \frac{z(z-n)^{n-1}}{n !} f^{(n)}(n),
$$

where it is assumed that (1.1) holds along the path of integration and that differentiation under the integral sign is permissible. This result was the basis of Abel's discovery of the series which now bears his name [1]. It would appear from his brief note that he believed (1.2) to hold for a wider class of functions than is actually the case, including $(z+x)^{-1}$ and $\log (z+x)$.

Conditions for the validity of (1.2) were first given by Halphen [2], who also noted that the Abel series for $(z+x)^{-1}$ represents an integral function, $G(z, x)$ say, which has several properties in common with $(z+x)^{-1}$. In this paper we find the asymptotic development of $G(z, 1)$ in the whole plane and show that in a certain region $G(z, 1)$ is asymptotically equivalent to $(z+1)^{-1}$. The asymptotic equivalence of an Abel series and its generating function holds, in a suitable domain, for a much wider class of functions than simple equivalence, and this part of the paper is a special case of a more general result recently given by Drs. A. J. and S. S. Macintyre [4].

2. Notation. $K$ is used to denote a positive number, not always the same at each occurrence, independent of the variable $z$, but possibly depending on other constants and parameters. 
The notation " $\psi(z)=O(\chi(z))$ " indicates a relation of the type " $|\psi|<K|\chi|$ for $|z|>K$ ", unless the contrary is stated.

The Abel series of $(z+1)^{-1}$ is

$$
G(z, 1)=G(z)=\sum_{0}^{\infty}(-1)^{n} \frac{z(z-n)^{n-1}}{(n+1)^{n+1}}
$$

and

$$
\begin{aligned}
G(z) & =\sum_{0}^{\infty}(-1)^{n} \frac{z(z-n)^{n-1}}{n !} \int_{0}^{\infty} \lambda^{n} e^{-(n+1) \lambda} d \lambda \\
& =\int_{0}^{\infty}\left\{\sum_{0}^{\infty}(-1)^{n} \frac{z(z-n)^{n-1}}{n !}\left(\lambda e^{-\lambda}\right)^{n}\right\} e^{-\lambda} d \lambda .
\end{aligned}
$$

The inversion of summation and integration is easily justified by uniform convergence. Consider now the Lagrange expansion of $e^{-z \lambda}$ in terms of $\lambda e^{-\lambda}$. This is

$$
e^{-z \lambda}=\sum_{0}^{\infty}(-1)^{n} \frac{z(z-n)^{n-1}}{n !}\left(\lambda e^{-\lambda}\right)^{n}
$$

and the ratio of two successive coefficients, for fixed $z$, gives

$$
\begin{aligned}
\left|\frac{z(z-n-1)^{n}}{(n+1) !}\right| \frac{z(z-n)^{n-1}}{n !} \mid & =\frac{n}{n+1}\left|\left(1-\frac{z-1}{n}\right)^{n} /\left(1-\frac{z}{n}\right)^{n-1}\right| \\
& =\frac{n}{n+1}\left|\exp \left\{n \log \left(1-\frac{z-1}{n}\right)-(n-1) \log \left(1-\frac{z}{n}\right)\right\}\right| \\
& =\frac{n}{n+1}\left|\exp \left\{1-\frac{1}{2 n}+O\left(\frac{1}{n^{2}}\right)\right\}\right| \\
& =e\left\{1-\frac{3}{2 n}+O\left(\frac{1}{n^{2}}\right)\right\}
\end{aligned}
$$

as $n \rightarrow \infty$. Hence, by Raabe's test, the expansion $(2.2)$ is valid in the region containing the origin and bounded by $\left|\lambda e^{-\lambda}\right|=1 / e$. This region will be denoted by $D$. The equation $\left|\lambda e^{-\lambda}\right| \leqslant 1 / e$ has been studied in detail by Hayes [3]. He has shown that if $\zeta=\lambda e^{-\lambda}$ then $|\zeta|=1 / e$ in the $\zeta$-plane corresponds to a curve having a node at $\lambda=1$ in the $\lambda$-plane. The loop of this curve bounds a region which we have called $D$. To $|\zeta|<1 / e$ correspond several domains in the $\lambda$-plane; one of these is $D$ and another includes the real axis from 1 to $\infty$. If $\lambda$ lies between 1 and $\infty$ 
then (2.2) converges to $\mu$, where $\mu$ is the unique correspondent of $\lambda$ that is contained in $D$ and lies on the real axis between 0 and 1 , i.e. $\lambda e^{-\lambda}=\mu e^{-\mu}$ with $1 \leqslant \lambda<\infty, 0<\mu \leqslant 1$. As $\lambda$ goes from 1 to $\infty$ then $\mu$ goes from 1 to 0 .

From the integral (2.1) we get

$$
\begin{aligned}
G(z)=\int_{0}^{1}\left\{\sum_{0}^{\infty}(-1)^{n} \frac{z(z-n)^{n-1}}{n !}\left(\mu e^{-\mu}\right)^{n}\right\} e^{-\mu} d \mu \\
+\int_{1}^{\infty}\left\{\sum_{0}^{\infty}(-1)^{n} \frac{z(z-n)^{n-1}}{n !}\left(\lambda e^{-\lambda}\right)^{n}\right\} e^{-\lambda} d \lambda,
\end{aligned}
$$

and when the variable in the second integral is changed from $\lambda$ to $\mu$ where $\lambda e^{-\lambda}=\mu e^{-\mu}$, the result is

$$
\begin{aligned}
G(z) & =\int_{0}^{1} e^{-(z+1) \mu}\left(\frac{1-\mu}{\lambda-1}\right) d \mu+\frac{1-e^{-(z+1)}}{z+1} \\
& =I(z+1)+\frac{1-e^{-(z+1)}}{z+1}, \quad z \neq-1 .
\end{aligned}
$$

The integral $\quad \int_{0}^{h}\left(\log _{2} \frac{1}{\mu}\right)^{r}\left(\log \frac{1}{\mu}\right)^{-s} e^{-z \mu} d \mu$,

where $h$ is small and fixed, is denoted by $I_{r, s}(z)$.

3. Statement of results. The complete expansion is included in the following :

Theorem 1.

$$
G(z)=J(z+1)+H(z+1)+\frac{1-e^{-(z+1)}}{z+1}
$$

in the region $|z|>K$ and $|\arg z| \leqslant 3 \pi / 2-\eta$, where $\eta$ is a fixed positive number less than $\pi / 4$.

The functions $J(z)$ and $H(z)$ are defined to be

$$
J(-z)=e^{z}\left\{\frac{1}{z}+\sum_{1}^{n} \frac{r ! c_{r}}{z^{r+1}}+O\left(\frac{1}{z^{n+2}}\right)\right\},
$$

where the $c_{r}$ are constants whose exact form will be given later, and

$$
\begin{aligned}
H(z)=\frac{1}{z \log z}-\frac{\log _{2} z}{z(\log z)^{2}} & +\frac{L_{1}+1}{z(\log z)^{2}}+\frac{\left(\log _{2} z\right)^{2}}{z(\log z)^{3}}-\frac{\left(3+2 L_{1}\right) \log _{2} z}{z(\log z)^{3}} \\
+ & \frac{1+3 L_{1}+L_{2}}{z(\log z)^{3}}-\frac{\left(\log _{2} z\right)^{3}}{z(\log z)^{4}}+O\left\{\frac{\left(\log _{2} z\right)^{2}}{z(\log z)^{4}}\right\}
\end{aligned}
$$


where $\log z$ is assumed to be real on the real positive axis, and

$$
L_{k}=\int_{0}^{\infty} e^{-t}(\log t)^{k} d t
$$

Theorem 1 follows from the three subsidiary theorems that follow, $I(z)$ being the integral defined by (2.3).

Theorem 2.

$$
I(z)=H(z)
$$

for

$$
-\frac{1}{2} \pi+\eta \leqslant \arg z \leqslant \frac{1}{2} \pi-\eta, \quad 0<\eta<\frac{1}{2} \pi .
$$

THEOREM 3.

$$
I(z)=J(z)
$$

for

$$
-\frac{1}{2} \pi+\eta \leqslant \arg (-z) \leqslant \frac{1}{2} \pi-\eta, \quad 0<\eta<\frac{1}{2} \pi .
$$

Theorem 4.

$$
I(z)=J(z)+H(z)
$$

for

$$
-\frac{1}{2} \pi+\eta \leqslant \arg z \leqslant \frac{1}{2} \pi-\eta
$$

and for

$$
-\frac{1}{2} \pi+\eta \leqslant \arg (-z) \leqslant \frac{1}{2} \pi-\eta, \quad 0<\eta<\frac{1}{4} \pi .
$$

In the region considered in Theorem $3, H(z)$ is negligible in relation to the remainder of $J(z)$, which is exponentially large so that in this region Theorem 1 follows from Theorem 3. Similar considerations show that Theorem 1 follows from Theorem 2 when the conditions of the latter theorem are satisfied. In the barrier region to which Theorem 4 applies, both $J(z)$ and $H(z)$ are relevant.

$H(z)$ is unlike the asymptotically small expansions usually found in asymptotic developments. It exhibits irregularity of structure and the decrease in order of successive terms is much smaller than normal.

4. Proof of Theorem 2. For the proof of Theorem 2 we require a series of lemmas.

LEMMA 1. $\lambda$ is an analytic function of $\mu$ in the region bounded by

$$
\left.\begin{array}{rl}
-\pi & =\phi-\rho \sin \phi, \phi \not \equiv-\pi \\
\pi & =\phi-\rho \sin \phi, \phi \neq \equiv \pi
\end{array}\right\},|\phi| \leqslant \pi, \mu=\rho e^{i \phi},
$$

except at the origin, where $\lambda e^{-\lambda}=\mu e^{-\mu}, \lambda \not \equiv \mu$ and $\lambda=1$ when $\mu=1$.

Consider the equation

$$
\zeta=s e^{-s}, s=r e^{i \theta} .
$$


Since $d \zeta / d s=(1-s) e^{-s}$, the possible singularities of $s$ as a function of $\zeta$ are $\zeta=1 / e$ with $s=1$ and $\zeta=0$ with $s=\infty$. Suppose that $\zeta$ encircles the origin $n$ times in the positive direction in the $\zeta$-plane and that $s=r_{1} e^{i \theta_{1}}$ and $s=r_{2} e^{i \theta_{2}}$ are the initial and final values of $s$. Then

$$
\theta_{1}-r_{1} \sin \theta_{1}=\theta_{2}-r_{2} \sin \theta_{2}-2 n \pi \text {. }
$$

Hence for each integer $n$, positive or negative, we get a different value of $s$, i.e. $\zeta=0$ is a logarithmic winding point of $s$. Near $s=1$ we have

$$
\zeta e=1-\frac{1}{2}(s-1)^{2}\{1+O(s-1)\}
$$

as $s \rightarrow 1$, and so at $s=1 \quad \zeta$ takes the value $1 / e$ with multiplicity 2 .

Consider two $\zeta$-sheets each cut along the negative real axis and along the positive real axis from $I$ to $\infty$. We take $-\pi \leqslant \arg \zeta \leqslant \pi$ in both, $-\pi \leqslant \arg (\zeta-1 / e) \leqslant \pi$ in the first and $\pi \leqslant \arg (\zeta-1 / e) \leqslant 3 \pi$ in the second. Denote by $\zeta_{1}$ and $\zeta_{2}$ points on the first and second of these sheets respectively. These two sheets are mapped on to the region in the $s$-plane bounded by

$$
\left.\begin{array}{rl}
-\pi & =\theta-r \sin \theta, \theta \neq \equiv-\pi \\
\pi & =\theta-r \sin \theta, \theta \neq \equiv \pi
\end{array}\right\},|\theta| \leqslant \pi,
$$

in a 1-1 manner. Let $\mu$ be a value of $s$ in the region defined by (4.1), other than $s=0$. If $\mu$ corresponds to $\zeta_{1}$, take $\lambda$ to be the point corresponding to $\zeta_{2}=1 / e+\left(\zeta_{1}-1 / e\right) e^{2 \pi i}$, and if $\mu$ corresponds to $\zeta_{2}$, take $\lambda$ to be the point corresponding to $\zeta_{1}=1 / e+\left(\zeta_{2}-1 / e\right) e^{-2 \pi i}$. This defines $\lambda$ as a function of $\mu$ in the region considered in the lemma. Also if $\mu \neq 0$ and $\mu \neq 1$ then $\zeta=\mu e^{-\mu}$ and $\zeta^{\prime}=\zeta e^{ \pm 2 \pi i}=\lambda e^{-\lambda}$, and since

$$
\frac{d \zeta}{d \mu}=(1-\mu) e^{-\mu}, \quad \frac{d \zeta^{\prime}}{d \lambda}=(1-\lambda) e^{-\lambda}, \quad \frac{d \zeta^{\prime}}{d \bar{\zeta}^{\prime}}=1
$$

it follows that

$$
\frac{d \lambda}{d \mu}=\frac{1-\mu}{1-\lambda} e^{\lambda-\mu}
$$

exists, and so $\lambda$ is a differentiable function of $\mu$. Hence $\lambda$ is an analytic function of $\mu$ in the region bounded by $-\pi=\phi-\rho \sin \phi$ and $\pi=\phi-\rho \sin \phi$, apart, possibly, from the points $\mu=0$ and $\mu=1$. Since $\lambda \rightarrow \infty$ as $\mu \rightarrow 0$ it follows that $\mu=0$ is a singular point of $\lambda . \quad \lambda$ is bounded in the neighbour. hood of $\mu=1$ and since $\mu=1$ is not a branch point of $\lambda$, as can be seen from the above, we see that $\lambda$ is analytic at $\mu=1$. From the construction of $\lambda$ and $\mu$ we have $\lambda e^{-\lambda}=\mu e^{-\mu}, \lambda \not \equiv \mu$ and $\lambda=1$ when $\mu=1$. This completes the proof of the lemma. 
Lemma 2. For $\lambda$ and $\mu$ real we have

$$
\begin{aligned}
\frac{1}{\lambda-1}=\frac{1}{\log (1 / \mu)}[1 & -\frac{\log _{2}(1 / \mu)}{\log (1 / \mu)}+\frac{1}{\log (1 / \mu)}+\frac{\left\{\log _{2}(1 / \mu)\right\}^{2}}{\{\log (1 / \mu)\}^{2}}-3 \frac{\log _{2}(1 / \mu)}{\{\log (1 / \mu)\}^{2}} \\
& \left.+\frac{1}{\{\log (1 / \mu)\}^{2}}-\frac{\left\{\log _{2}(1 / \mu)\right\}^{3}}{\{\log (1 / \mu)\}^{3}}+O\left\{\frac{\left\{\log _{2}(1 / \mu)\right\}^{2}}{\{\log (1 / \mu)\}^{3}}\right\}\right]
\end{aligned}
$$

as $\mu \rightarrow 0$.

$\lambda$ is found in terms of $\mu$ by successive approximation as follows. Since $\lambda e^{-\lambda}=\mu e^{-\mu}$ it follows that

$$
\begin{aligned}
\lambda & =\log (1 / \mu)+\log \lambda+\mu \\
& =\log (1 / \mu)+O\left\{\log _{2}(1 / \mu)\right\}
\end{aligned}
$$

as $\mu \rightarrow 0$. By continuing this process we finally get

$$
\lambda=\log (1 / \mu)+\log _{2}(1 / \mu)+\frac{\log _{2}(1 / \mu)}{\log (1 / \mu)}-\frac{1}{2} \frac{\left\{\log _{2}(1 / \mu)\right\}^{2}}{\{\log (1 / \mu)\}^{2}}+O\left\{\frac{\log _{2}(1 / \mu)}{\{\log (1 / \mu)\}^{2}}\right\},
$$

and a simple application of the binomial theorem gives (4.4).

Lemma 3. When $|\arg z| \leqslant \pi / 2-\eta, 0<\eta<\pi / 2$, then for $|z|>k>1$

$$
\begin{aligned}
I_{r, s}(z)= & \frac{\left(\log _{2} z\right)^{r}}{z(\log z)^{s}} \sum_{\nu=0}^{r} \frac{1}{\left(\log _{2} z\right)^{\nu}} \\
& \times\left\{a_{0}^{r, 8, \nu}+\frac{L_{1} a_{1}^{r, s, \nu}}{\log z}+\ldots+\frac{L_{n} a_{n}^{r, s, \nu}}{(\log z)^{n}}+O\left(\frac{1}{(\log z)^{n+1}}\right)\right\},
\end{aligned}
$$

where $\log z$ and $\log _{2} z$ are real for real positive $z>1$ and $a_{n}^{r_{s} s, v}=(-1)^{\nu}\left(\begin{array}{c}r \\ v\end{array}\right) b_{n}^{s, v}$ with $b_{n}^{8, v}$ the coefficient of $x^{n}$ in the expansion of

$$
\left(x+\frac{x^{2}}{2}+\ldots+\frac{x^{n}}{n}+\ldots\right)\left\{1-\left(-\frac{s}{1}\right) x+\ldots+(-1)^{n}\left(-\frac{s}{n}\right) x^{n}+\ldots\right\}
$$

in ascending powers of $x$.

Take $z \mu=t . \quad$ Then

$$
I_{r, 8}(z)=\frac{1}{z} \int_{0}^{z h}\left(\log _{2} \frac{z}{t}\right)^{r}\left(\log \frac{z}{t}\right)^{-s} e^{-t} d t
$$

where the integration is along the line $\arg t=\arg z$. Now

$$
\begin{aligned}
\left(\log _{2} \frac{z}{t}\right)^{r} & =\left\{\log _{2} z+\log \left(1-\frac{\log t}{\log z}\right)\right\}^{r} \\
& =\sum_{\nu=0}^{r}\left(\begin{array}{c}
r \\
\nu
\end{array}\right)\left(\log _{2} z\right)^{r-\nu}\left\{\log \left(1-\frac{\log t}{\log z}\right)\right\}^{\nu} \\
& =\sum_{\nu=0}^{r}(-1)^{\nu}\left(\begin{array}{c}
r \\
\nu
\end{array}\right)\left(\log _{2} z\right)^{r-v}\left\{\frac{\log t}{\log z}+\ldots+\frac{1}{p}\left(\frac{\log t}{\log z}\right)^{p}+R_{p}\right\}^{\nu}
\end{aligned}
$$


where

$$
R_{p}=\left(\frac{\log t}{\log z}\right)^{p+1} \int_{0}^{1} \frac{(1-u)^{n}}{\{1-(\log t / \log z) \bar{u}\}^{n+\overline{1}+1}} d u .
$$

Also $\left(\log \frac{z}{t}\right)^{-s}=\frac{1}{(\log z)^{s}}\left(1-\frac{\log t}{\log z}\right)^{-s}$

$$
=\frac{1}{(\log z)^{s}}\left\{1-\left(\begin{array}{c}
-s \\
1
\end{array}\right) \frac{\log t}{\log z}+\ldots+(-1)^{q}\left(\begin{array}{c}
-s \\
q
\end{array}\right)\left(\frac{\log t}{\log z}\right)^{q}+R_{q}{ }^{\prime}\right\}
$$

where

$$
R_{q}^{\prime}=(-1)^{q+1}(q+1)\left(\begin{array}{c}
-s \\
q+1
\end{array}\right)\left(\frac{\log t}{\log z}\right)^{q+1} \int_{0}^{1}(1-u)^{q}\left(1-\frac{\log t}{\log z} u\right)^{-s-q-1} d u .
$$

Using these expression in $I_{r, s}(z)$, we have

$$
\begin{aligned}
I_{r, s}(z)= & \frac{1}{z} \int_{0}^{z h} \sum_{\nu=0}^{r}(-1)^{\nu} \frac{(\log 2)^{r-\nu}}{(\log z)^{s}}\left\{\frac{\log t}{\log z}+\ldots+\frac{1}{p}\left(\frac{\log t}{\log z}\right)^{p}+R_{p}\right\}^{\nu} \\
& \times\left\{1-\left(\begin{array}{c}
-s \\
1
\end{array}\right) \frac{\log t}{\log z}+\ldots+(-z)^{q}\left(\begin{array}{c}
-s \\
q
\end{array}\right)\left(\frac{\log t}{\log z}\right)^{q}+R_{q}^{\prime}\right\} e^{-t} d t \\
= & \frac{1}{z} \frac{\left(\log _{2} z\right)^{r}}{(\log z)^{s}} \sum_{\nu=0}^{r}\left(\frac{1}{\log _{2} z}\right)^{\nu} \\
& \times \int_{0}^{z h}\left\{a_{0}^{r, s, \nu}+a_{1}^{r, s, \nu} \frac{\log t}{\log z}+\ldots+a_{n}^{r, s, \nu}\left(\frac{\log t}{\log z}\right)^{n}+S_{n}\right\} e^{-t} d t .
\end{aligned}
$$

To obtain the above expression $p$ and $q$ have been taken sufficiently large to ensure that the first $n$ terms of the product of the bracketed expressions coincide with the first $n$ terms of the expression given in $x$, with

$$
\frac{\log t}{\log z}=x,
$$

in the statement of the lemma. Hence it follows that $S_{n}$ is a sum of terms of the type

$$
A R_{p}^{B} R_{q}^{\prime C}\left(\frac{\log t}{\log z}\right)^{D}
$$

with $A, B, C$ and $D$ constants. The following conditions are satisfied by $A, B, C$ and $D$ :

(i) $B, C$ and $D$ are non-negative integers;

(ii) $n \leqslant \min (p, q)$;

(iii) $B+C \geqslant 1$ when $D \leqslant n$. 
The first of these conditions is obvious, the second can be satisfied by taking $p$ and $q$ large enough and the third is true since if $B+C=0$ and $D \leqslant n$ then the corresponding term contributes to the sum of the first $n$ terms.

To complete the proof of Lemma 3 we require Lemmas 4 and 5.

Lemma 4. When $|\arg z| \leqslant \pi / 2-\eta$,

$$
\int_{0}^{2 h} e^{-t}(\log t)^{k} d t=L_{k}+O\left(e^{-\epsilon|z|}\right), \quad \epsilon>0 .
$$

Lemma 5. When $|\arg z| \leqslant \pi / 2-\eta$ and $\log z$ is real for real positive $z$ then

$$
\int_{0}^{2 h} e^{-t} S_{n} d t=O\left\{\left(\frac{1}{\log z}\right)^{n+1}\right\}
$$

Proofs of Lemmas 4 and 5. By an application of Cauchy's theorem we find, noting that $|\arg z| \leqslant \pi / 2-\eta$,

which is Lemma 4.

$$
\begin{aligned}
\int_{0}^{2 h} e^{-t}(\log t)^{k} d t & =\int_{0}^{|z| h} e^{-t}(\log t)^{k} d t+O\left(e^{-\varepsilon|z|}\right) \\
& =\int_{0}^{\infty} e^{-t}(\log t)^{k} d t+O\left(e^{-\mathrm{e}|z|}\right) \\
& =L_{k}+O\left(e^{-\epsilon|z|}\right)
\end{aligned}
$$

To prove Lemma 5 we have to show that

$$
\int_{0}^{z h} R_{p}^{B} R_{q}^{\prime C}\left(\frac{\log t}{\log z}\right)^{D} e^{-t} d t=O\left\{\left(\frac{1}{\log z}\right)^{n+1}\right\}
$$

when $B+C \geqslant 1$ if $D \leqslant n$. If $B+C=0$ then (4.6) is obvious, and so we assume that $B+C \geqslant 1$. For $|t| \leqslant|z|^{1 / 2}$ we have

$$
\left|\frac{\log t}{\log z}\right| \leqslant\left|\left(\frac{1}{2} \log |z|+i \arg z\right)(\log z)^{-1}\right| \leqslant \frac{1}{2}+\epsilon, \quad 0<\epsilon<\frac{1}{2}
$$

for $|z|$ sufficiently large. Hence

$$
\begin{aligned}
\left|R_{p}\right| & \leqslant\left|\frac{\log t}{\log z}\right|^{p+1} \int_{0}^{1} \frac{(1-u)^{p}}{\left(1-\frac{1}{2} u-\epsilon u\right)^{p+1}} d u \\
& =O\left\{\left(\frac{\log t}{\log z}\right)^{p+1}\right\} .
\end{aligned}
$$


Similarly, for $|t| \leqslant|z|^{1 / 2}$

$$
\left|R_{q}^{\prime}\right|=O\left\{\left(\frac{\log t}{\log z}\right)^{q+1}\right\} .
$$

In the range $|z|^{1 / 2} \leqslant|t| \leqslant|z| h$

Hence

$$
\begin{aligned}
\left|\frac{\log t}{\log z}\right| & =\left|\frac{\log |t|+i \arg z}{\log z}\right| \\
& =\left|1-\frac{\log |z / t|}{\log z}\right| \leqslant 1-\frac{K}{|\log z|} .
\end{aligned}
$$

$$
\left|R_{p}\right| \leqslant\left|\frac{\log t}{\log z}\right|^{p+1} \int_{0}^{1}\{1-u+(k /|\log z|)\}^{p+1} d u=O\left\{(\log t)^{p+1}\right\}
$$

Similarly, for $|z|^{1 / 2} \leqslant|t| \leqslant|z| h$

$$
\left|R_{q}^{\prime}\right|=O\left\{(\log t)^{q+1}\right\} \text {. }
$$

We now consider the integral of (4.6) with the range of integration divided into the two sub-ranges considered above, and use (4.7) and $(4.8)$ in the first part and (4.9) and (4.10) in the second part. We get

$$
\begin{aligned}
& \int_{0}^{z h} R_{p}{ }^{B} R_{q}^{\prime C}\left(\frac{\log t}{\log z}\right)^{D} e^{-t} d t=\left(\int_{0}^{z|z|^{-1 / 2}}+\int_{z|z|^{-1 / 2}}^{z h}\right) R_{p}^{B} R_{q}^{\prime C}\left(\frac{\log t}{\log z}\right)^{D} e^{-t} d t \\
&= O\left\{\left(\frac{1}{\log z}\right)^{B(p+1)+C(q+1)+D}\right\}+O\left(e^{-\epsilon|z|)}\right. \\
& \quad+O\left\{\left(\frac{1}{\log z}\right)^{D} \int_{|z|^{1 / 2}}^{\infty}(\log t)^{B(p+1)+C(q+1)+D} e^{-t} d t\right\} \\
&=O\left\{\left(\frac{1}{\log z}\right)^{n+1}\right\} .
\end{aligned}
$$

LEMmA 6. We have for $|\arg z| \leqslant \frac{1}{2} \pi-\eta, 0<\eta<\frac{1}{2} \pi$,

$$
\left.\int_{0}^{h} \mu\left(\log _{2} \frac{1}{\mu}\right)^{r}\left(\log \frac{1}{\mu}\right)^{-s} e^{-z \mu} d \mu=O \frac{\left(\log _{2} z\right)^{r}}{\mid z^{2}(\log z)^{s}}\right\}
$$

Let $z \mu=t$, giving

$$
\begin{aligned}
\int_{0}^{h} \mu\left(\log _{2} \frac{1}{\mu}\right)^{r} & \left(\log \frac{1}{\mu}\right)^{-s} e^{-z \mu} d \mu \\
& =\frac{1}{z^{2}} \int_{0}^{z \hbar}\left(\log _{2} \frac{z}{t}\right)^{r}\left(\log \frac{z}{t}\right)^{-s} t e^{-t} d t=O\left\{\frac{\left(\log _{2} z\right)^{r}}{z^{2}(\log z)^{s}}\right\}
\end{aligned}
$$

by Lemma 3 , since a factor $t$ in the integrand leaves the order of the integral unaffected. The functions $\log z$ and $\log _{2} z$ are assumed to be real for $z$ real and greater than 1 . 
We now proceed to the proof of Theorem 2 itself. We have

$$
I(z)=\int_{0}^{1} e^{-z \mu} \frac{1-\mu}{\lambda-1} d \mu=\left(\int_{0}^{h}+\int_{h}^{1}\right) e^{-z \mu} \frac{1-\mu}{\lambda-1} d \mu .
$$

From the proof of Lemma 1 it is seen that $\left|\frac{1-\mu}{\lambda-1}\right|$ is bounded for $h \leqslant \mu \leqslant 1$, and so

$$
\int_{h}^{1} e^{-z \mu} \frac{1-\mu}{\lambda-1} d \mu=O\left(e^{-(|z|)}\right)
$$

By Lemmas 2 and 6

Hence

$$
\int_{0}^{h} e^{-z \mu} \frac{\mu}{\lambda-1} d \mu=O\left(\frac{1}{z^{2} \log z}\right)
$$

$$
\begin{aligned}
I(z)=\int_{0}^{h} \frac{e^{-z \mu}}{\lambda-1} d \mu+O\left(\frac{1}{z^{2} \log z}\right)+O\left(e^{-\mathrm{e}|z|}\right) \\
=I_{0,1}(z)-I_{1,2}(z)+I_{0,2}(z)+I_{2,3}(z)-3 I_{1,3}(z) \\
\quad+I_{0,3}(z)-I_{3,4}(z)+O\left\{I_{2,4}(z)\right\}+O\left(\frac{1}{z^{2} \log z}\right) .
\end{aligned}
$$

When Lemma 3 is applied to the integrals in (4.12), Theorem 2 follows.

\section{Proof of Theorem 3.}

Lemma 7. For $|\mu-1|<1, \lambda-1=\sum_{1}^{\infty} b_{n}(\mu-1)^{n}$, where the $b_{n}$ are constants which can be calculated.

This is an immediate consequence of Lemma 1 and the fact that $\lambda=1$ when $\mu=1$. It can be shown that $b_{1}=-1$.

In the circle $|\mu-1|<1, \lambda-1$ has only one simple zero, which is at $\mu=1$. Hence for $|\mu-1|<1$ it follows that

$$
\frac{1-\mu}{\lambda-1}=-\left\{\sum_{1}^{\infty} b_{n}(\mu-1)^{n}\right\}^{-1}=\sum_{0}^{\infty} c_{n}(\mu-1)^{n}
$$

where the $c_{n}$ can be calculated in terms of the $b_{n}$. Since $b_{1}=-1$ it is seen that $c_{0}=1$.

Assume now that the conditions of Theorem 3 hold. Set $-z=y$ and we get

$$
\int_{0}^{1} e^{y \mu} \frac{1-\mu}{\lambda-1} d \mu=\left(\int_{0}^{1-h}+\int_{1-h}^{1}\right) e^{y \mu} \frac{1-\mu}{\lambda-1} d \mu
$$


From Lemma $2, \frac{1-\mu}{\lambda-1}$ is bounded in $(0,1-h)$, and so

$$
\int_{0}^{1-h} e^{y \mu} \frac{1-\mu}{\lambda-1} d \mu=O\left(e^{(1-\imath)|y|}\right), \quad 0<\epsilon<1 .
$$

Putting $1-\mu=\sigma$ in the second integral in (5.2) and using (5.1) we have

$$
I(z)=I(-y)=e^{y}\left[\int_{0}^{h} e^{-\sigma \nu}\left\{1+\sum_{1}^{n} c_{r} \sigma^{r}+O\left(\sigma^{n+1}\right)\right\} d \sigma+O\left(e^{-e|y|}\right)\right] .
$$

The range $(0, h)$ may be replaced by $(0, \infty)$ since the error introduced is $O\left(e^{(1-)): y \mid}\right)$ and

$$
O\left(e^{(1-\epsilon)|y|}\right)=O\left\{e^{\operatorname{Re} y} \int_{0}^{h} e^{-\sigma \operatorname{Re} y} \sigma^{n+1} d \sigma\right\} .
$$

Hence Theorem 3 follows.

6. Proof of Theorem 4. In the following we assume $z=\xi^{e^{\frac{1 \pi}{\pi}}}$ where $-\eta \leqslant \arg \xi \leqslant \eta$ with $0<\eta<\frac{1}{4} \pi$, so that $z$ is confined to a sector enclosing the positive imaginary axis. The proof for $z$ in a sector enclosing the negative imaginary axis is similar and is omitted. The proof depends on the integration of $e^{-z \mu} \frac{1-\mu}{\lambda-1}$ round the contour $\Gamma$ indicated in the diagram ${ }^{1}$ where $1>k_{1}>\tan \eta$. The following preliminary lemmas are required.

LEMMA 8. $\frac{1-\mu}{\lambda-1}$ is regular in and on $\Gamma$.

This follows immediately from Lemma 1 .

Lemma 9. Provided $1>k_{1}>\tan \eta$,

$$
\int_{-k_{1} i}^{1-k_{1}^{i}} e^{-z \mu} \frac{1-\mu}{\lambda-1} d \mu=O\left(e^{-\epsilon|z|}\right), \quad \epsilon>0 .
$$

Let $\mu+k_{1} i=u$. Then

$$
\begin{aligned}
\int_{-k_{1} i}^{1-k_{1} i} e^{-z \mu} \frac{1-\mu}{\lambda-1} d \mu & =\int_{0}^{1} e^{-z\left(u-k_{1} i\right)} \frac{1-\left(u-k_{1} i\right)}{\lambda\left(u-k_{1} i\right)-1} d u \\
& =O\left(e^{\operatorname{Re}\left(z k_{1} i\right)} \int_{0}^{1} e^{-u \operatorname{Re} z} d u\right) \\
& =O\left\{|z|^{-1} \exp \left(-|z|\left(k_{1} \cos \eta-\sin \eta\right)\right)\right\}
\end{aligned}
$$

and the last expression is $O\left(e^{-\epsilon|z|}\right)$ provided $k_{1} \cos \eta-\sin \eta>0$.

1 See p. 144. 
LemMA 10. For $\frac{1}{2} \pi-\eta \leqslant \arg z \leqslant \frac{1}{2} \pi+\eta, 0<\eta<\frac{1}{4} \pi$ and $1>k_{1}>\tan \eta$

Set $\mu=-u i$ and

$$
\int_{0}^{-k_{1} i} e^{-z \mu} \frac{1-\mu}{\lambda-1} d \mu=H(z)
$$

$$
\int_{0}^{-k_{1} i} e^{-z \mu} \frac{1-\mu}{\lambda-1} d \mu=\frac{1}{i} \int_{0}^{k_{1}} e^{i z u} \frac{1+i u}{\lambda(-u i)-1} d u .
$$

If $(\lambda(-u i)-1)^{-1}$ is expressed in terms of $u$ in a similar manner to that of Lemma 2 we find that

$$
\begin{aligned}
(\lambda(-u i)-1)^{-1}= & \frac{1}{\log (1 / u)}\left[1-\frac{\log _{2}(1 / u)}{\log (1 / u)}-\frac{\frac{1}{2} \pi i-1}{\log (1 / u)}+\frac{\left\{\log _{2}(1 / u)\right\}^{2}}{\{\log (1 / u)\}^{2}}\right. \\
& +(i \pi-3) \frac{\log _{2}(1 / u)}{\{\log (1 / u)\}^{2}}+\left\{-\frac{i \pi}{2}+\left(\frac{i \pi}{2}-1\right)^{2}\right\} \frac{1}{\{\log (1 / u)\}^{2}} \\
& \left.-\frac{\left\{\log _{2}(1 / u)\right\}^{3}}{\{\log (1 / u)\}^{3}}+O\left\{\frac{\left\{\log _{2}(1 / u)\right\}^{2}}{\{\log (1 / u)\}^{3}}\right\}\right]
\end{aligned}
$$

Replacing $z$ by $\xi e^{\frac{1}{2} \pi i}$ and integrating, we have

$$
\begin{aligned}
\int_{0}^{-k_{1} i} & e^{-z \mu} \frac{1-\mu}{\lambda-1} d \mu=\frac{1}{i}\left[\frac{1}{\xi \log \xi}-\frac{\log _{2} \xi}{\xi(\log \xi)^{2}}+\frac{L_{1}+1-\frac{1}{2} \pi i}{\xi(\log \xi)^{2}}+\frac{\left(\log _{2} \xi\right)^{2}}{\xi(\log \xi)^{3}}\right. \\
& +\frac{\left\{(i \pi-3)-2 L_{1}\right\} \log _{2} \xi}{\xi(\log \xi)^{2}}+\frac{L_{1}+L_{2}+2 L_{1}\left(1-\frac{1}{2} \pi i\right)+\left\{-\frac{1}{2} \pi i+\left(\frac{1}{2} \pi i-1\right)^{2}\right\}}{\xi(\log \xi)^{3}} \\
& -\frac{\log _{2} \xi}{\xi(\log \xi)^{4}}+O\left\{\frac{\left(\log _{2} \xi\right)^{2}}{\xi(\log \xi)^{4}}\right\}
\end{aligned}
$$

When the right-hand side of $(6.3)$ is expressed in terms of $z$ it is seen to be $H(z)$.

Lemma 11. For $\frac{1}{2} \pi-\eta \leqslant \arg z \leqslant \frac{1}{2} \pi+\eta, 0<\eta<\frac{1}{4} \pi$ and $1>k_{1}>\tan \eta$,

$$
\int_{1-k_{1} i}^{1} e^{-z \mu} \frac{1-\mu}{\lambda-1} d \mu=J(z)
$$

Setting $u=-i(1-\mu)$ gives, with $z=\xi e^{\frac{1 \pi i}{n}}$,

$$
\begin{array}{r}
\int_{1-k_{1} i}^{1} e^{-z \mu} \frac{1-\mu}{\lambda-1} d \mu=i \int_{0}^{k_{1}} e^{-z(1-u i)} \frac{u i}{\lambda(1-u i)-1} d u \\
=e^{-z} \int_{0}^{k_{1}} e^{-\xi u}\left\{1+\sum_{1}^{n} c_{r} e^{t r \pi i} u^{r}+O\left(u^{n+1}\right)\right\} d u
\end{array}
$$

from Lemma 7. The right-hand side of $(6.4)$ becomes $J(z)$ when expressed in terms of $z$. 
LEMMA 12. If $\gamma$ is the arc $|\mu|=k,-\frac{1}{2} \pi \leqslant \arg \mu \leqslant 0$, then

as $k \rightarrow 0$.

$$
\int_{\gamma} e^{-z \mu} \frac{1-\mu}{\lambda-1} d \mu \rightarrow 0
$$

From Lemma 1 we see that $\left|\frac{1-\mu}{\lambda-1}\right|$ is bounded on $\gamma$ as $k \rightarrow 0$, whence follows $(6.5)$.

If we now integrate $e^{-z \mu} \frac{1-\mu}{\lambda-1}$, where $z$ satisfies the conditions of Theorem 4, round $\Gamma$, we get

$$
\left(\int_{k}^{1}+\int_{1}^{1-k_{1} i}+\int_{1-k_{1} i}^{-k_{1} i}+\int_{-k_{1} i}^{-k i}+\int_{\gamma}\right)^{-z \mu} \frac{1-\mu}{\lambda-1} d \mu=0
$$

where it assumed that $1>k_{1}>\tan \eta$. Letting $k \rightarrow 0$ and using Lemma 12 we have

$$
\int_{0}^{1} e^{-z \mu} \frac{1-\mu}{\lambda-1} d \mu=\left(\int_{0}^{-k_{1} i}+\int_{-k_{1} i}^{1-k_{1} i}+\int_{1-k_{1} i}^{1}\right) e^{-z \mu} \frac{1-\mu}{\lambda-1} d \mu .
$$

Theorem 4 follows by using Lemmas 9,10 and 11 .

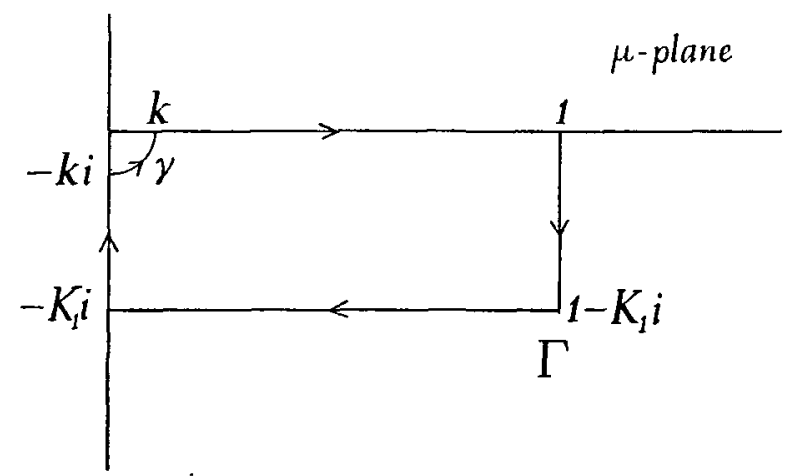

In conclusion I should like to thank Dr. A. J. Macintyre for suggesting to me the topic of this paper and for his guidance throughout its preparation.

\section{REFERENCES.}

1. N. H. Abel, Oeuvres d'Abel (1881), t. 2, 67-81.

2. G. H. Halphen, "Sur une série d'Abel ", Bull. de la Soc. Math. (1881-1882), 67.

3. N. D. Hayes, "Roots of the transcendental equation associated with a certain difference-differential equation ", Journal London Math. Soc., 25 (1950), 226-232.

4. A. J. Macintyre and S. S. Macintyre, "Theorems on the convergence and asymptotic validity of Abel's series", Proc. Roy. Sac. Edinburgh, A, 63 (1952), 222-231.

\section{University College of North Staffordshire,}

KEELE. 